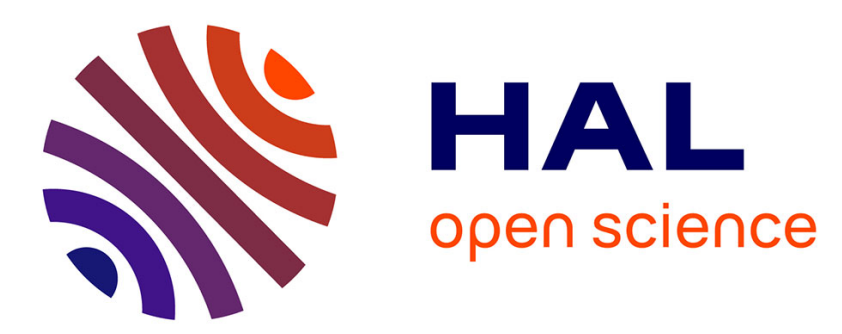

\title{
Optimized distribution of a large number of power electronics components cooled by conjugate turbulent natural convection
}

\author{
Antoine Baudoin, Didier Saury, Cecilia Boström
}

\section{To cite this version:}

Antoine Baudoin, Didier Saury, Cecilia Boström. Optimized distribution of a large number of power electronics components cooled by conjugate turbulent natural convection. Applied Thermal Engineering, 2017, 124, pp.975-985. 10.1016/j.applthermaleng.2017.06.058 . hal-02134976

\author{
HAL Id: hal-02134976 \\ https://hal.science/hal-02134976
}

Submitted on 19 Apr 2020

HAL is a multi-disciplinary open access archive for the deposit and dissemination of scientific research documents, whether they are published or not. The documents may come from teaching and research institutions in France or abroad, or from public or private research centers.
L'archive ouverte pluridisciplinaire HAL, est destinée au dépôt et à la diffusion de documents scientifiques de niveau recherche, publiés ou non, émanant des établissements d'enseignement et de recherche français ou étrangers, des laboratoires publics ou privés. 
A. Baudoin, D. Saury, C. Boström, Optimized distribution of a large number of power electronics components cooled by conjugate turbulent natural convection, Applied Thermal Engineering, vol. 124, pp. 975-985 (2017) doi: http://dx.doi.org/10.1016/j.applthermaleng.2017.06.058

\title{
Optimized distribution of a large number of power electronics components cooled by conjugate turbulent natural convection
}

\author{
Antoine Baudoin ${ }^{\mathrm{a}, *}$, Didier Saury ${ }^{\mathrm{b}}$, Cecilia Boström $^{\mathrm{a}}$ \\ ${ }^{a}$ Division for Electricity, Uppsala University, Box 534, 75121 Uppsala SWEDEN \\ ${ }^{b}$ Institut PPRIME, CNRS, ENSMA, Université de Poitiers, BP 40109, F-86961 Futuroscope Chasseneuil CEDEX, France
}

\begin{abstract}
Natural convection allows for passive cooling which is used in many engineering applications. Placing dissipating components on a common vertical heatsink can be optimized to give the best possible cooling capacity. In this study, a numerical model for three-dimensional conjugated convective and conductive heat transfer was used to evaluate the distribution of up to 36 flush-mounted rectangular heaters. The temperature profiles and the heat fluxes were compared with experimental data for validation. The dissipated power was set as an input parameter and the optimal distribution was selected as the one with the lowest temperature elevation. Two different heuristics - a geometric parameter and an artificial neural network - were proposed and evaluated as alternatives to heavy CFD calculations.
\end{abstract}

Keywords: Optimization, discrete heating, conjugate natural convection, artificial neural network, Computational Fluid Dynamic

\section{Nomenclature}

$\alpha \quad$ Thermal diffusivity

$\beta \quad$ Volumetric thermal expansion coefficient

\section{$\rho \quad$ Density}

$\lambda_{\text {fluid }} \quad$ Fluid thermal conductivity

$\lambda_{\text {plate }} \quad$ Plate thermal conductivity

$\lambda_{1}, \lambda_{2} \quad$ Geometric parameter

$\lambda_{3} \quad$ Geometric parameter

$\nu \quad$ Kinematic viscosity

$\mu \quad$ Viscosity

$\mu_{T} \quad$ Turbulent viscosity

$\phi_{i j} \quad$ Angle between sources number

$\theta$ Dimensionless temperature $\Delta T /\left(T_{\max }-T_{\infty}\right)$

$C_{p} \quad$ Heat capacity

$d_{i j} \quad$ Distance between sources

e Plate thickness

F Sum of external forces

$g$ Gravitational constant

$h \quad$ Convective heat transfer coefficient

I Identity matrix

$k \quad$ Turbulence kinetic energy

$L \quad$ Heat source width

$p \quad$ Pressure

$q " \quad$ Heat flux density

$Q \quad$ Volume heat source

$H \quad$ Heat source height

$N \quad$ Number of heat sources

$N u \quad$ Nusselt number $\partial \theta / \partial Y^{*}$

\section{Prandtl number}

$R a \quad$ Rayleigh number

$T \quad$ Temperature

u Velocity

$Y^{*} \quad$ Dimensionless distance to the wall

$z \quad$ Vertical coordinate

$Z^{*} \quad$ Dimensionless vertical coordinate

$(=z / H)$

$\mathrm{W} / \mathrm{mK}$

$\mathrm{m}^{1-\bar{b}} \quad \Delta Z$

$\mathrm{m}^{1-b}$

$\Delta Z$

Vertical spacing

$\Delta T$

$\mathrm{kg} / \mathrm{ms}$

$\mathrm{kg} / \mathrm{ms}$

$\mathrm{rad}$

Temperature elevation $T-T_{\infty}$

Artificial Neural Network

BL

CFD

Boundary Layer

Computational Fluid Dynamic

\section{Introduction}

$\mathrm{J} / \mathrm{kgK}$

$\mathrm{m}$

$\mathrm{m}$

$\mathrm{N} / \mathrm{m}^{3}$

$\mathrm{m} / \mathrm{s}^{2}$

$\mathrm{W} / \mathrm{m}^{2} \mathrm{~K}$

$\mathrm{m}^{2} / \mathrm{s}^{2}$

$\mathrm{m}$

$\mathrm{N} / \mathrm{m}^{2}$

$\mathrm{W} / \mathrm{m}^{2}$

$\mathrm{W} / \mathrm{m}^{3}$

$\mathrm{m}$

Natural convection occurs spontaneously every time a temperature gradient appears in a fluid. As such, it is a very reliable cooling technique. In addition to reliability, passive convective cooling is also quiet and cheap. A better understanding of this phenomena is crucial in many engineering applications and it has been extensively studied [1].

Passive cooling, which is enabled by free convection, is well suited for electronic packages. But this technique offers limited cooling capacity in air and has primarily been limited to low power applications [2]. However, because of its high level of reliability natural convection cooling is also interesting in power generation, some example of which are solar photovoltaic panels [3] and power electronics for marine energy for which liquid cooling is easily available

* Corresponding author 
[4]. The majority of the literature considers Prandtl number, $\mathrm{Pr}$, around 0,7 which is typical for air. Although air cooling is more common because of obvious simplicity of implementation, liquid cooling can be preferred to reach higher heat flux, either by direct cooling with dielectric fluid or by indirect cooling with water [5]. In the case of direct liquid cooling, the cooled components are immersed in the liquid. However, the components are more often flush-mounted on a plate, whose opposite side is in contact with the coolant, making the cooling indirect. The later liquid cooling technique has a poorer thermal conductance because of the solid-solid and solid-liduid interfaces $[6,7]$.

A lot of studies have been conducted on uniform heating or uniform temperature conditions, which are the most simple cases [8]. However, most of engineering applications involve discrete heat sources, such as electronic or power components. In such cases, conduction in the substrate and convection in the fluid are two competing phenomena [9]. Problems involving heat sources found in the literature can be categorized into open cavities [10], channels $[3,11,12]$, enclosures $[13,14,15]$, and open boundaries [16].

Like all kind of flows, buoyancy-driven flows can be either fully laminar or present a transition toward turbulence. In case of natural convection, the transition is governed by the Rayleigh number, $R a$, equivalent to the Reynolds number. It is still not fully understood and it remains difficult to predict [17]. In the case of discrete sources, micro electronics packaging has been the driver for the research work on natural convection. Most of the studies deal thus with small dimensions and therefore low Rayleigh numbers and laminar flows. However, turbulence may have a significant influence on heat transfers [3].

Natural convection lead to complex flow fields and nonuniform heat transfer. Regular distributions of the heat sources lead therefore to disparities in the temperature at different components [6]. Placing dissipating components on a common heatsink is not trivial if the best possible cooling is to be achieved from natural convection. As regular spacing has been shown to give only suboptimal results [16], optimization is interesting in case of limited available space.

The interest for heat source array grew with miniaturization in microelectronics and the consequent increase in heat flux densities. In 1995, an array of identical discrete heat sources was studied numerically and experimentally with conjugated conduction and natural convection [13]. The case of protruding electronic components was treated numerically in [18], where the influence of the spacing was investigated. In the last decade, numerous attempts have been made to optimize different cooling systems with nonuniform heating and discrete heat sources. The constructal theory introduced by Bejan [16, 19], gives a frame for approaching these kind of problems. It stated that to be reliable a system "must evolve in such a way that it provides easier access to the imposed currents that flow through it".
The currents can be heat fluxes when considering the design of cooling solutions. In general the cooling considered is optimized when the maximum temperature (i.e. hot spots) is minimized. This corresponds to configurations for which the temperature is equal at each heat source $[16,20]$.

Most of the attempts to find the optimal configuration for discret heating restrict the optimization problem in one direction (i.e. one space coordinate is varied). Optimal locations for heaters defined by the distance to leading edge was investigated with a 2-dimensional model in [16]. More recently, the source heat flux was otpimized as a piecewise-constant one-dimensional function in [20, 21] for laminar internal flows.

Experiments have also been conducted for band heaters in [22], where the natural convective cooling of discrete heater sources in an enclosure was studied. The nonuniform configuration was confirmed to give higher cooling coefficient and velocity field measurements were performed.

Although reliability of components is largely determined by working temperature and thus proper thermal management, Computational Fluid Dynamic (CFD) might not be cost efficient in all engineering applications as it requires large computational resources. A five-sources problem was treated in $[23,24,25]$, using a heuristic approach to propose an alternative to exhaustive CFD parametric studies.

Genetic algorithms have been used to optimize the heat flux [26, 27, 28, 29]. These evolutionary techniques are often associated to Artificial Neural Network (ANN) $[24,25,28,30]$. In [30] a neural network was trained in order to optimize the placement of 5 heat sources on a $5 \times 5$ positions grid. It was showed that the temperature calculated with the ANN was in good agreement with the CFD model. For such an optimization problem there exist many near optimum solutions. It is also possible to optimize a configuration with fixed unequally-heated elements, like in [31], where the heat duty among 15 heat sources mounted on a wall of a duct has been optimized. A array of $6 \times 6$ electronic devices on a heatsink was cooled by forced convection in [32].

However, the complexity of the optimization problem increases rapidly with the number of heat sources to position in a three-dimensional configuration [29]. Optimization work have often been conducted for a limited number, and only few examples can be found for more than 10 heaters [23]. Alternatively, the passive cooling capacity can be enhanced with optimized heat sink design [33].

Reliability issues in offshore devices (due to expansive maintenance operation) make these renewable power systems a new important application of thermal engineering (e.g. for cables [34] or for generators [35]). Power electronics play a major role in the development of renewable energy sources, for instance wind power, solar power, and marine power. The present study addresses the need, in power conversion systems for marine energy, to find an 


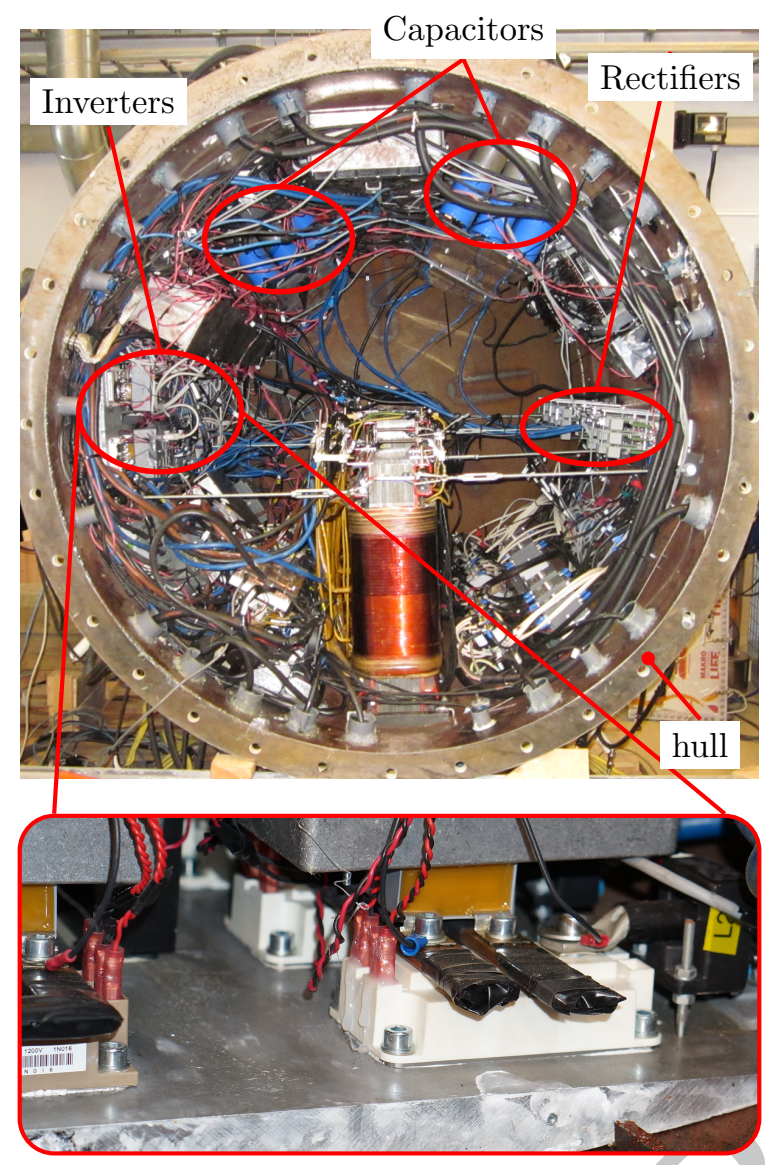

Figure 1: Inside a submerged substation prototype for wave power farms. The components are mounted on the inner face of the pressurized vessel and are passively cooled by conjugate natural convection.

optimal positioning of dissipating components as shown in Figure 1 (similarly to pins position optimization in [36]). However, the results are generally valid for any similar distribution of heat sources on a plate cooled by natural convection. This work differs from the previously discussed studies by combining a large number of sources, threedimensionality, liquid cooling and a turbulent model. The aim is to optimize the positioning of multiple and identical heat sources on a vertical plate with respect to cooling considerations.

A high number of sources together with the possibility lead to numerous possible solutions, almost impossible to test with an exhaustive approach. Extensive CFD investigation for an optimal solution is therefore not reasonable. CFD can only be used for a set of a limited number of calculation for training of ANN or for validation of a found optimal solution.

\section{Method}

The case studied consists of flush-mounted rectangular heat sources, to be placed on a conductive plate with a fixed area of $1 \times 2 \mathrm{~m}$. This conductive heatsink plate was $1 \mathrm{~cm}$ thick and has a conductivity of $240 \mathrm{~W} / \mathrm{Km}$ (i.e. typically aluminium). The total dissipated power was imposed as uniform over the heat sources. It was ramped up from $10 \mathrm{~W}$ to $8 \mathrm{~kW}$, which corresponded to heat flux density up to $140 \mathrm{~kW} / \mathrm{m}^{2}$. Several approaches were tried with the aim of comparing the possible distribution of the heat sources in terms of temperature elevation. The fluid used in the model is water and the dimension of the heat sources $(10 \times 5 \mathrm{~cm})$, which matches the typical dimensions of power electronic components. However generic heaters have been considered. Only steady-state temperature was studied.

In electrical systems, three-phase is the standard and it requires a multiple of three numbers of modules (e.g. diodes, IGBTs...). Therefore, the cases treated in this article were chosen with a number of sources, $N$, multiple of 3 and up to 36 .

Distributions of 12, 24 and 36 heat sources were tested (see Figure 3 for an example for 36 heaters). A set of distributions (number 1 to 6 ) was first defined with uniform vertical and horizontal spacings. Regular and staggered grids were used for a varying number of rows (as suggested in [36]). Then, the vertical spacing was calculated as an increasing function of the height. The spacing was first depending on the height as an arithmetic sequence with a common difference set to cover the largest area on the plate (number 7 to 11 ). For distributions number 12 to 18 the spacing was increasing with the height in the power of $1 / 4$.

The Rayleigh number is defined in Equation 1. The length, $L$, used in this equation, is the total height covered by heat sources.

$$
R a_{L}=\frac{g \cdot \beta \cdot \Delta T \cdot L^{3}}{\alpha \cdot \nu}
$$

\subsection{Numerical scheme}

The commercial finite-element software, Comsol multiphysics ${ }^{1}$ was chosen to perform numerical calculations. The governing equations are presented in Equations 2 to 4. Figure 2 shows the boundary conditions implemented in the model. The open boundary was verified to be sufficiently from the wall not to perturb the boundary layer. Thermal and flow periodic conditions were set on the side walls (in order to represent to the cylindrical shape of the hull of the pressurized vessel). The Boussinesq approximation is not used due to the large dimensions of the geometry.

$$
\rho C_{p} \mathbf{u} \cdot \nabla T+\nabla \cdot(-\lambda \nabla T)=Q
$$

$$
\begin{aligned}
& \rho(\mathbf{u} \cdot \nabla) \mathbf{u}=\nabla \cdot\left[-p \mathbf{I}+\left(\mu+\mu_{T}\right)\left(\nabla \mathbf{u}+(\nabla \mathbf{u})^{T}\right)\right. \\
& \left.-\frac{2}{3}\left(\mu+\mu_{T}\right)(\nabla \cdot \mathbf{u}) \mathbf{I}-\frac{2}{3} \rho k \mathbf{I}\right]+\mathbf{F}
\end{aligned}
$$

\footnotetext{
${ }^{1}$ Comsol Multiphysics 4.4, Comsol Inc., MA 01803, USA.
} 


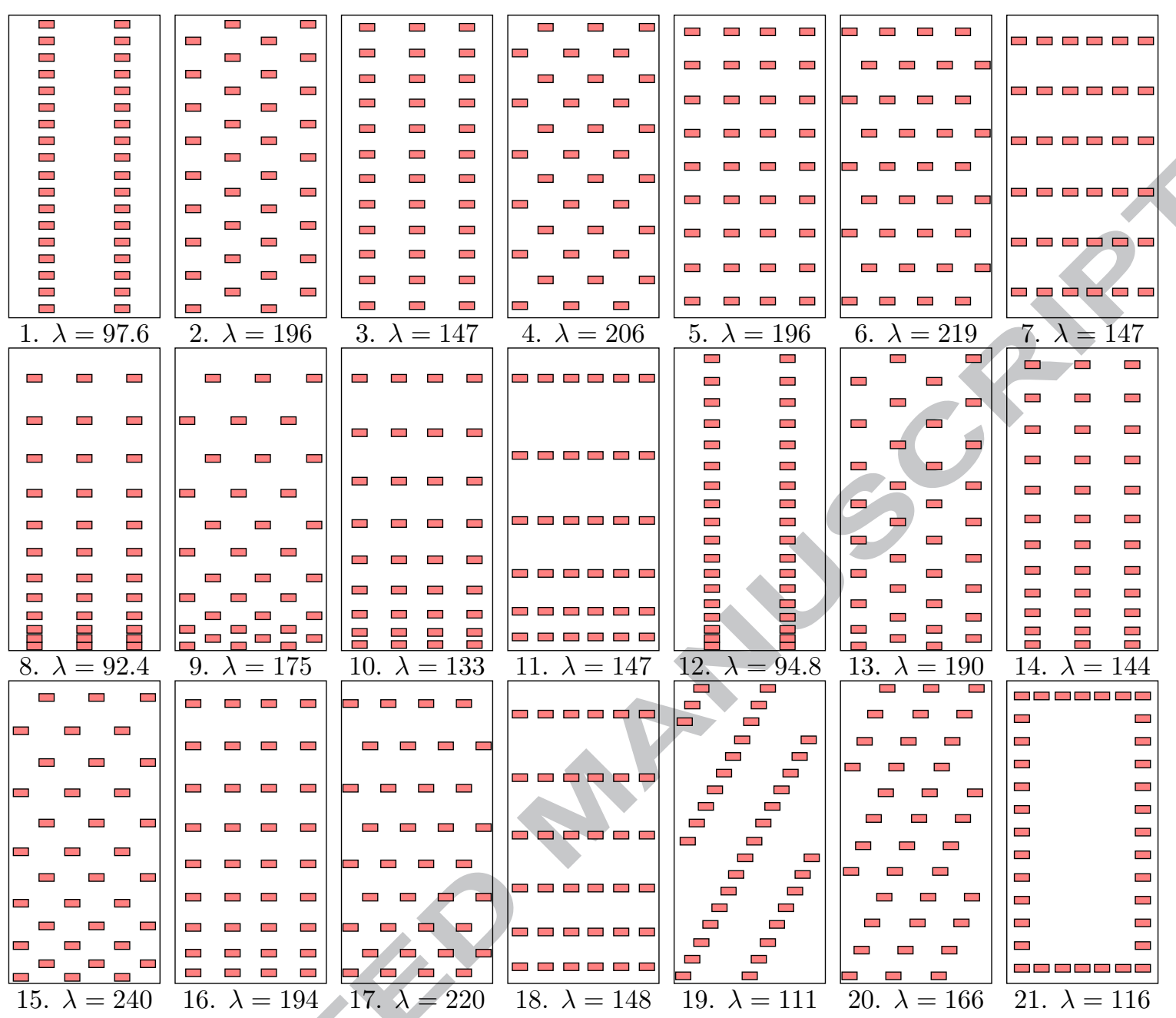

Figure 3: First guess distributions for 36 heat sources. $\lambda$ is the geometric parameter defined in Equation 8.

$$
\nabla \cdot(\rho \mathbf{u})=0
$$

A mesh sensitivity study was performed to ensure meshindependent results. The meshes were constituted by 14 flat boundary-layer mesh elements on the vertical wall (approximately $0.2 \mathrm{~mm}$ thick) and 8 at the bottom wall. The mesh was refined by a factor of 2 around the heat sources.

In order to account for turbulence effects, the SST (Menter's Shear Stress Transport) turbulence model was used. This model combines the $\mathrm{k}-\omega$ and $\mathrm{k}-\epsilon$ turbulence models such that the first one is used in the inner region of the boundary layer. The radiative heat transfer were neglected given the low temperature difference and the IR-opaque fluid. In the experiment, a low emissivity material was chosen to limit the radiative effects. The side boundaries were set to be periodic thermal and kinetic conditions, so the results can remain valid for cylindrical shapes or wider dimensions.

\subsection{Experimental setup}

The results from the model were compared with experimental data for a 9 heat sources rectangular distribution (i.e. a $3 \times 3$ array). The details of the experimental setup can be found in $[37,38]$. A K-type thermocouple of diameter $12,7 \mu \mathrm{m}$ was used to measured temperature in the air flow (the ambient temperature was measured with a PT100 sensor immersed in water and the data were acquired with a multimeter NI PXI 1031 installed in a NI $4071 \mathrm{DMM}$ unit). The uncertainty was below $0,5 \mathrm{~K}$. The thermocouple was mounted on a multi-axes linear servo actuators system for movement in the z-vertical and ydirection. In the direction normal to the plate (y axis), the precision of the moving axis was $\pm 0.02 \mathrm{~mm}$. In this way, five measurement points were taken within the first millimeter. It was shown to be sufficient to obtain a high correlation factor for the linear regression $\left(\mathrm{r}^{2}>0.99\right)$ to determine the Nusselt number $\left(\partial \theta / \partial Y^{*}\right)$.

For convenience, the laboratory setup was placed in air. Printed copper boards, with a known electrical resistance, were used as heat source. The experimental and numerical data were compared at similar Rayleigh number. Besides, for conjugated conduction and natural convection in a plate, the width of the temperature peak is estimated by $\sqrt{\lambda_{\text {plate }} e / h}[7]$. Therefore, the quantity, $A$, 


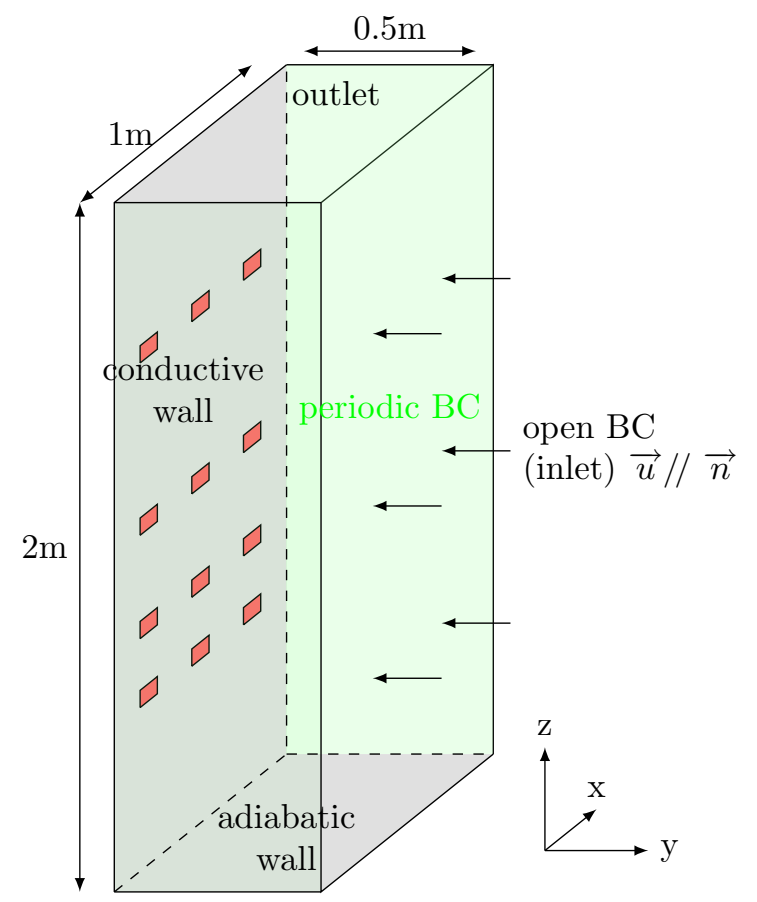

Figure 2: Schematic view of CFD model. The gray surfaces are noslip wall boundary condition. The green ones are periodic boundary conditions (both side wall). The conductive wall is covered with boundary heat sources (in red) and the backside is set as an adiabatic boundary.

shown in Equation 5 was equal in air and water conditions for comparison of profiles in the plate.

$$
A=\frac{\lambda_{\text {plate }} e}{\lambda_{\text {fluid }} R a^{1 / 4}}
$$

\subsection{Heuristic methods}

\subsubsection{Geometric parameter}

As suggested in several works, an heuristic approach can be adopted to efficiently find near-optimal solutions for the optimization problem, by using a geometric parameter (see Equation 6). Good correlation was found between this unique parameter and CFD calculations or experimental results $[23,24,25]$.

$$
\lambda_{1}=\frac{\sum_{i} d_{i}^{2}}{1+z_{c}^{2}}
$$

However, the above parameter tends to favour distributions with sources on the boundaries of the domain. This is valid for small number of sources and small dimensions of the plate. For numerous sources and large plates, this method will leave unused the cooling capacity in the middle of the plate and is thus unlikely to limit the maximum temperature. Conductive cross-coupling can be reduced by using a new proposed parameter shown in Equation 7. It is defined as the generalized mean of the distance between the heat sources.

$$
\lambda_{2}=\left(\sum_{\forall j \neq i} d_{i j}^{-1 / a}\right)^{-a}
$$

where $a$ is a positive parameter which can be tuned with the results from the CFD model. The parameter $\lambda_{2}$ decreases toward the minimum of $d_{i j}$ when $a$ increases. An other parameter was defined and tested as shown in Equation 8. Introducing the $z$-coordinate in the formula will favour larger spacing in the upper part of the plate where the cooling is less efficient.

$$
\lambda_{3}=\min _{\forall j \neq i}\left(\frac{d_{i j}}{z_{i}^{b}}\right)
$$

where $b$ is also a parameter which can be tuned with the results from the CFD model. It is expected that the higher $\lambda_{2}$ and $\lambda_{3}$, the lower the temperature. The correlation between these proposed parameters and the temperature elevation was studied to investigate the possibility for predicting the temperature with such a geometric parameter.

\subsubsection{Neural Network}

Artificial Neural Network (ANN) have been used to solve optimization problem for heat sources on vertical plate cooled by natural conduction $[24,30]$.

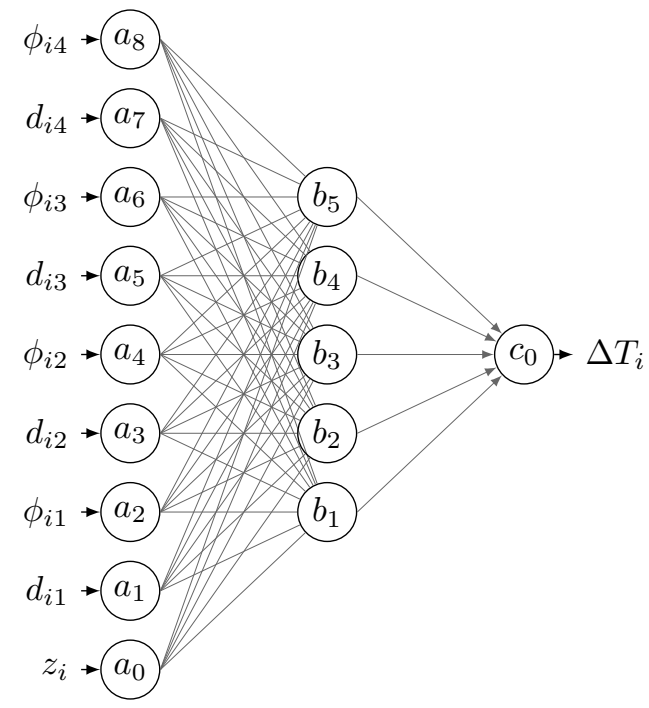

Figure 4: Example of neural network for the heat source number $i$ and with 4 surrounding heat sources. $d_{i 1}$ is the distance between the source number $i$ and its closest neighbour. $\Delta T_{i}$ is the temperature at the heat source number $i . \quad \phi_{i j}$ is the angle of the line between sources number $i$ and $j . a_{i}, b_{i}$ and $c_{0}$ are neurons in the input layer, in the hidden layer and in the output layer respectively.

The neural network ${ }^{2}$ shown in Figure 4 includes one hidden layer. The input layer had a number of neurons equal to twice the number of heat sources. The output layer had one neuron corresponding to the temperature

\footnotetext{
${ }^{2}$ pybrain v0.31 library for Python
} 
of one heat source. It was found to be more efficient than evaluating a whole distribution directly as it was performed in [30]. The feed-forward back-propagation algorithm with momentum and with bias was used. The results from the CFD model were used to train the network. $20 \%$ of the numerical data were kept as a validation dataset and was not considered for the training.

The ANN in this paper calculates the temperature for one heat source at a time, given its environment (i.e. relative position of the surrounding heat sources). The position of the neighbours was given as input parameters in form of cylindrical coordinates. The neighbours were ordered by decreasing distance. The use of cartesian coordinates resulted in poorer ability of the network. One hidden layer with biases was implemented. An increase of the number of input layers or the number of neurons in these layers gave no improvement on the performance of the network.

\section{Results}

\subsection{CFD model validation}

As it can be seen in Table 1, the results were shown to be mesh-independent. The meshes were constituted by 14 flat boundary-layer mesh elements on the vertical wall (approximately $0.2 \mathrm{~mm}$ thick) and 8 at the bottom wall. The mesh was refined by a factor of 2 around the heat sources.

\begin{tabular}{ccc}
\hline Mesh elements & Temperature & Change \\
\hline 137219 & 20.278 & - \\
359565 & 20.396 & $0.58 \%$ \\
616765 & 20.384 & $0.06 \%$ \\
\hline
\end{tabular}

Table 1: CFD grid dependency for uniform heating.

The results from the CFD model have been compared with the experimental data. The profiles are presented in Figures 5 and 6 along the middle vertical line. The comparison was made for two extreme values of the vertical spacing to confirm that the model reproduced the influence of this parameter. The Rayleigh number was between $10^{9}$ and $10^{11}$. The reference position is the middle row. The local comparison of the wall temperature shows good agreement between numerical and experimental results (see Figure 5). The amplitude of the spatial temperature variations are well reproduced by the CFD model. The temperature decrease downstream of the heat sources is slightly slower in the CFD model.

The heat transfer are described with the local Nusselt number. Figure 6 shows that numerical results matches the experimental data. The most noticeable difference is again downstream of the heat sources and between the heat sources with a large spacing. The heat transfers were observed to be inverted in these regions (i.e. the plate is heated by the fluid). However, the Nusselt number remains relatively low in absolute value and it does not affect
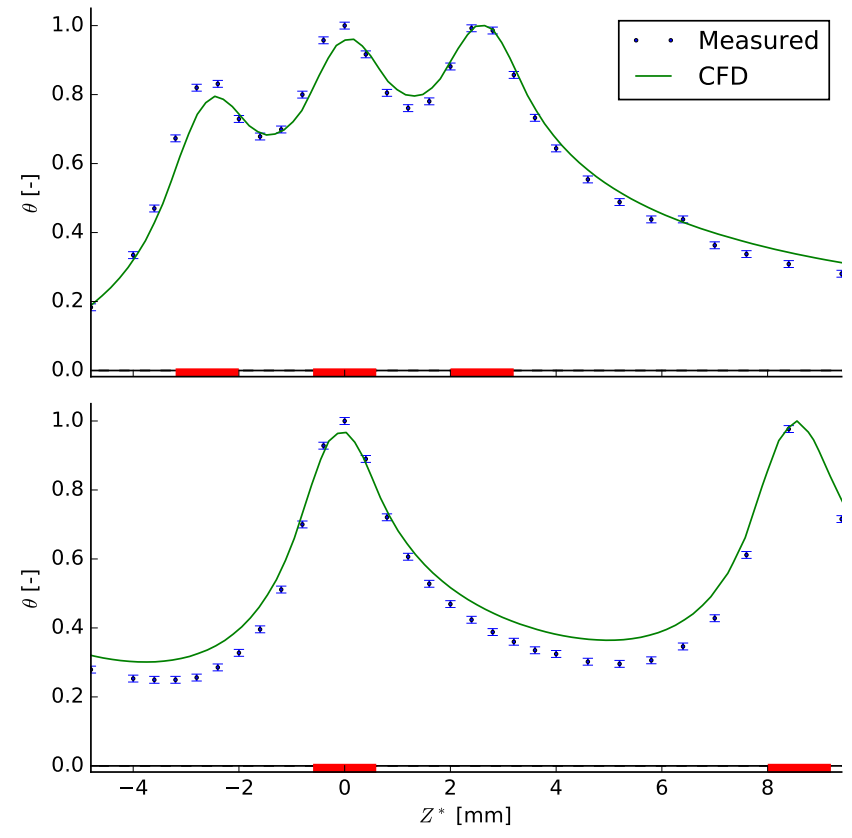

Figure 5: Comparison of CFD- and measured- dimensionless wall temperature profile. The vertical spacing was set to $13 \mathrm{~cm}$ (top) and $43 \mathrm{~cm}$ (bottom). The red lines show the position of the heat sources.

the ability of the model to describe the thermal interactions between the heat sources. In addition, the experimental Nusselt number is calculated by linear regression over the temperature points close to wall and the accuracy in limited for a low slope.

\subsection{Comparison of distributions}

The temperature for the warmest heat source was extracted from all CFD calculations. This was the criterion to evaluate the quality of the cooling corresponding to each distribution. The results are shown in Figure 7 for different numbers of heat sources. The Rayleigh number varied from $2 \cdot 10^{10}$ to $6 \cdot 10^{12}$.

In the case of 12 heat sources, the heat sources covered only a limited share of the heatsink plate (i.e. only $3 \%$ of the area). The distribution is only loosely constrained by the thermal efficiency objective. Many distributions can be considered as optimal. The plate is large enough so that the heat sources can be slightly shifted without affecting the temperature.

For 24 and 36 heat sources, the best distributions are number 15 and 17, which are staggered configurations with 3 and 4 heaters per rows respectively. The best cooling is obtained when the vertical is non-uniform and varies proportionally to $z^{1 / 4}$. This observation is in good agrrement with earlier results indicating better cooling when the heat sources are more tightly packed around the leading edge [16]. The temperature field in the vertical plate is presented in Figure 9 for two optimal distributions.

In Figure 8, the temperature per row is shown for all distributions with 36 heat sources in 9 rows. The staggered 

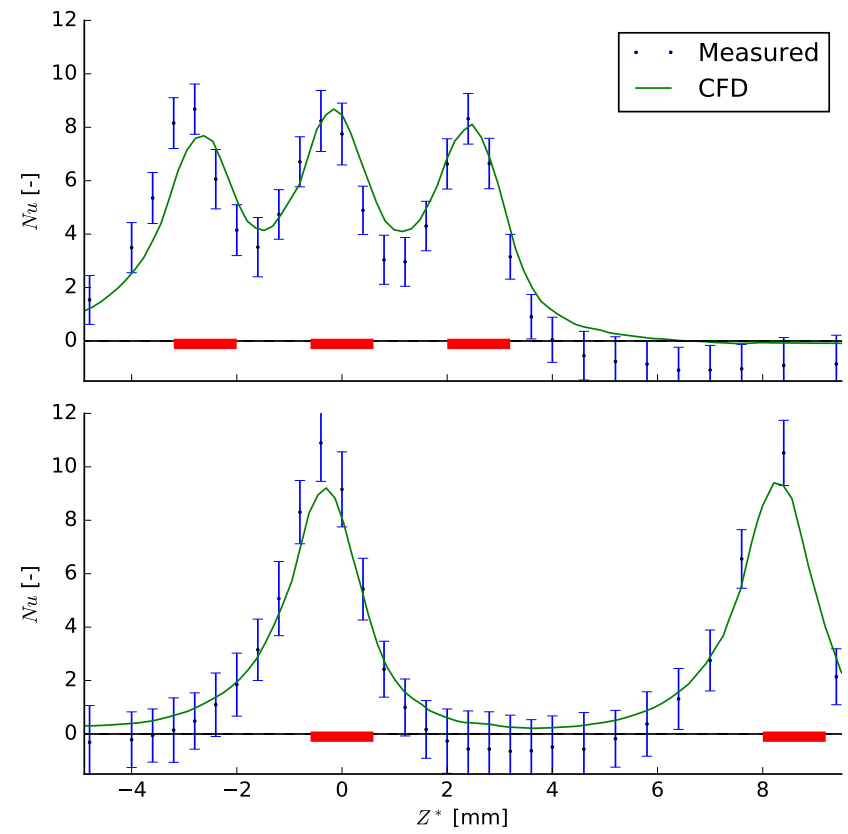

Figure 6: Comparison of CFD- and measured- Nusselt number. The vertical spacing was set to $13 \mathrm{~cm}$ (top) and $43 \mathrm{~cm}$ (bottom). The red lines show the position of the heat sources.

distributions performed better both for a rectangular distribution with uniform spacing and for a varying spacing. For a uniform spacing, the temperature increases almost constantly because the cooling at upper rows is degraded by the convective flow from the lower heat sources. On the contrary, the curves for the varying spacing presented a plateau, because this effect was compensated with a larger spacing. The maximum temperature is therefore lower for these configurations.

For a low dissipated power (Figure 8 - top), the temperature is almost constant over the rows with an increase spacing. This observation indicates that this distribution is optimal. For a higher power, the temperature starts decreasing in the upper part of the heatsink plate. This can be explained by the transition to a turbulent regime. When the flow becomes turbulent, the Nusselt number is proportional to $R a^{1 / 3}$ [39]. This yields an optimal heat flux to be uniform.

\subsection{Predictive methods}

Finally, the accuracy of two heuristic methods were evaluated. The aim was to predict the temperature elevation at very low computational costs. Comparison with the results from the CFD model is thus presented in the following section. In Figures 10 to 12 each point represents one tested configuration.

\subsubsection{Geometric parameter}

The CFD results were used to tune the parameters $a$ and $b$ (see equation 7 and 8). In Figure 10, it can be seen that the parameter $\lambda_{2}$ gave a good accuracy over the entire
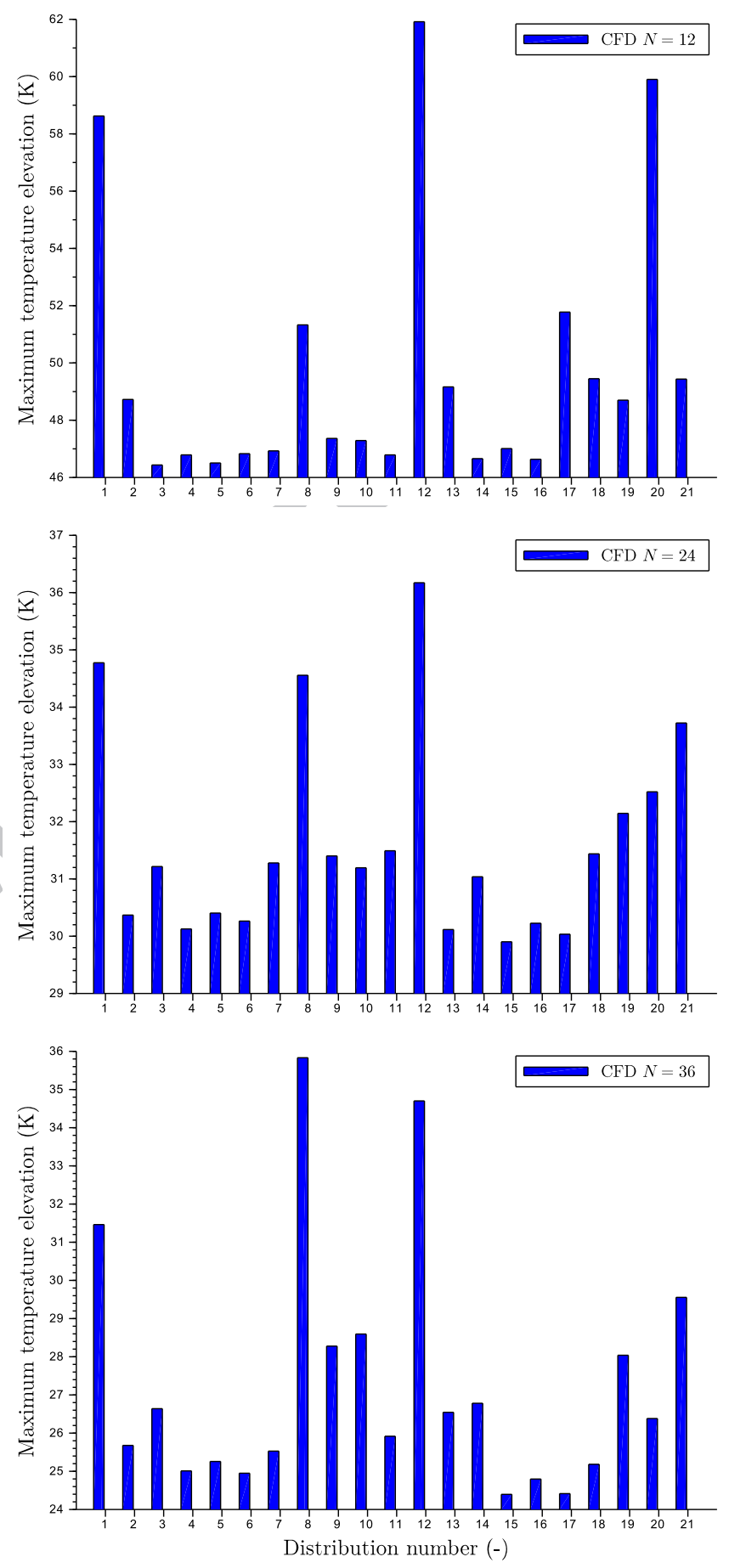

Figure 7: Comparison of the maximum temperature elevation for 12 (top), 24 (middle) and 36 (bottom) heat sources for a total dissipated power of $8 \mathrm{~kW}$.

range of tested distribution. The parameter $a$ was found to be optimal around 3 .

The parameter $\lambda_{3}$ appeared to give results in better agreement with the temperature modelled by CFD. As it can be seen in Figure 11, the error was below 3\% for the temperature predicted based on this geometric parameter. This conclusion is valid for 24 and 36 heat sources. Table 

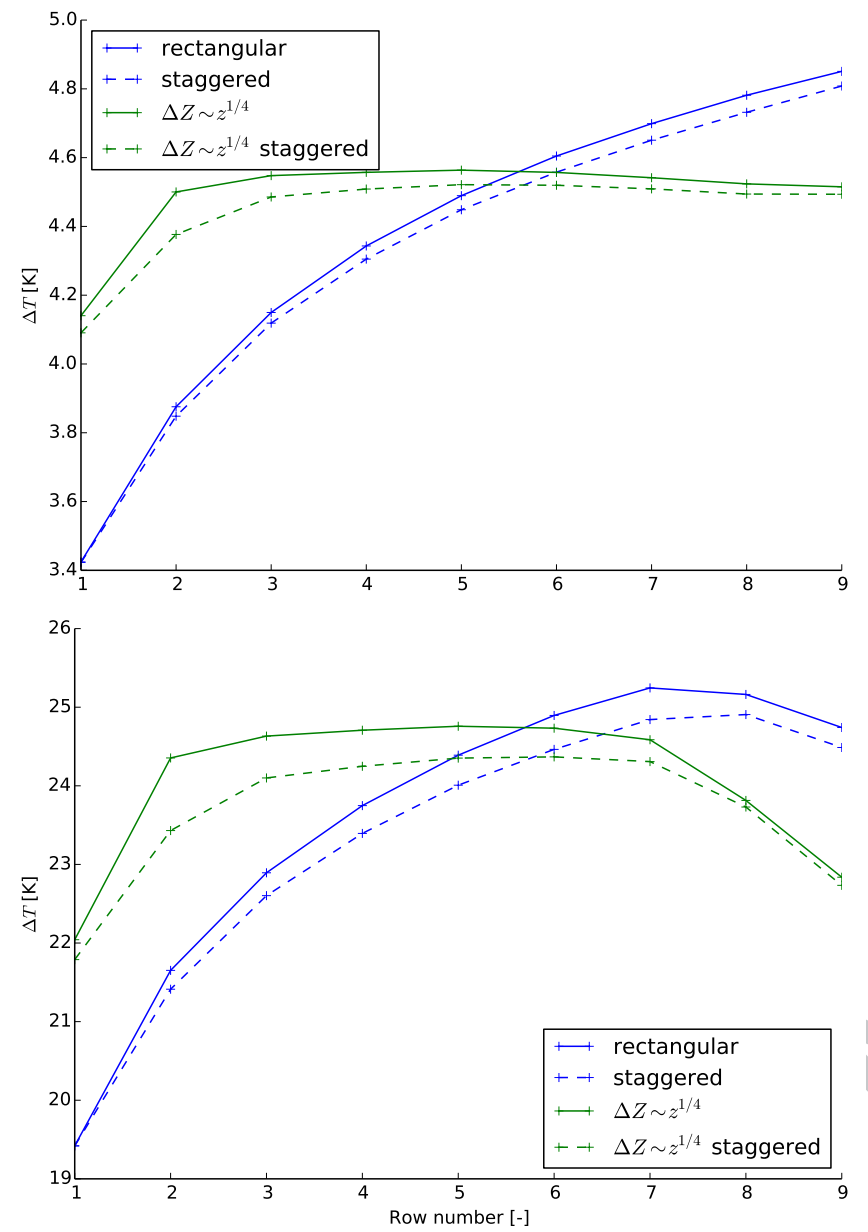

Figure 8: Mean temperature per row for 36 heat sources. Distributions number 5, 6, 16 and 17 . Total dissipated power of $1 \mathrm{~kW}$ (top) and $8 \mathrm{~kW}$ (bottom). The Rayleigh number was $2 \cdot 10^{10}$ and $6 \cdot 10^{11}$ respectively.

\begin{tabular}{ccc}
\hline Power $(\mathrm{kW})$ & $N=24$ & $N=36$ \\
\hline 1 & 0.24 & 0.29 \\
2 & 0.2 & 0.243 \\
4 & 0.2 & 0.148 \\
8 & 0.094 & 0.145 \\
\hline
\end{tabular}

Table 2: Optimal parameter $b$.

2 shows how the optimal parameter $b$ decreases with the dissipated power. This is due to the transition of the flow to a turbulent regime as described in the Section 3.2.

\subsubsection{Neural network}

The ANN was trained with the results from the CFD model. The RMS error on the validation dataset was monitored. The error was decreasing until about 1000 epochs and overfitting was observed after that. The training was performed repeatedly and the best of the networks was selected. The trained networks can be used directly for other distributions of heat sources on a vertical plate.

After training, the network could successfully predict
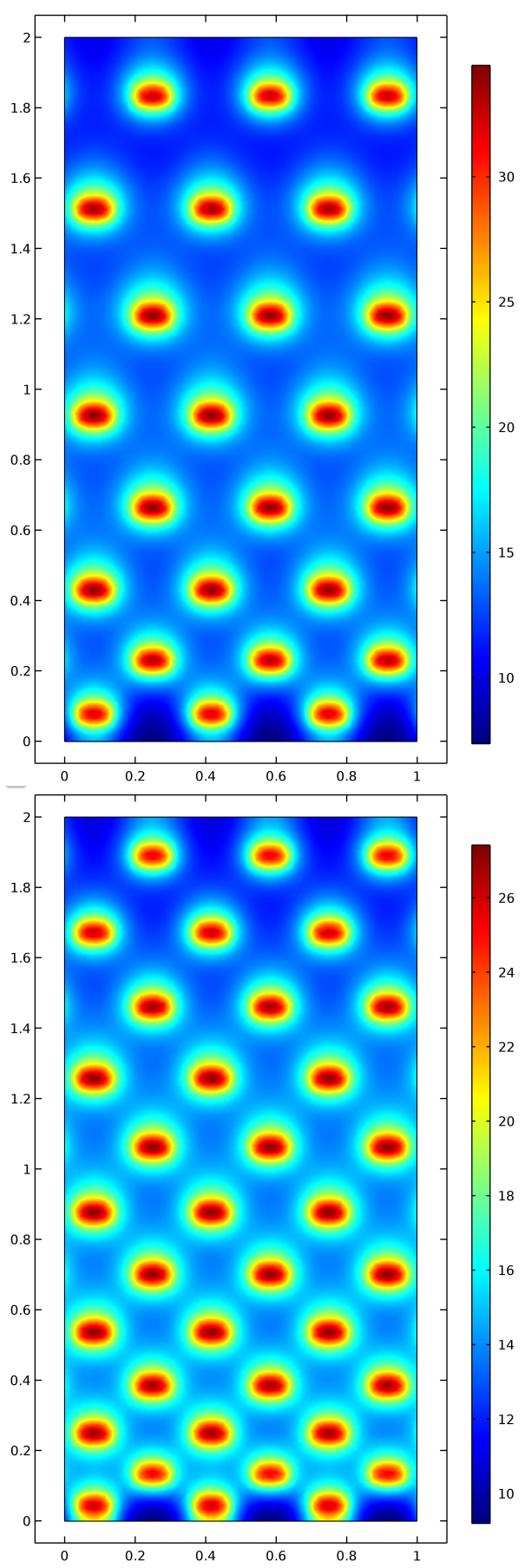

Figure 9: Temperature elevation in the heatsink plate for optimal distributions of 24 (top) and 36 (bottom) heat sources. Total dissipated power of $8 \mathrm{~kW}$.

the temperature of each heat source. In Figure 12, the error given by the ANN compared to the CFD model was below $5 \%$ for most of the distributions. When considering only the distributions giving the lowest temperature, the accuracy is better. It is partly due to the higher density of training data in this region. For optimization purposes, it is the ability of the ANN to distinguish the cooling performance of the near optimal solutions which is crucial. 

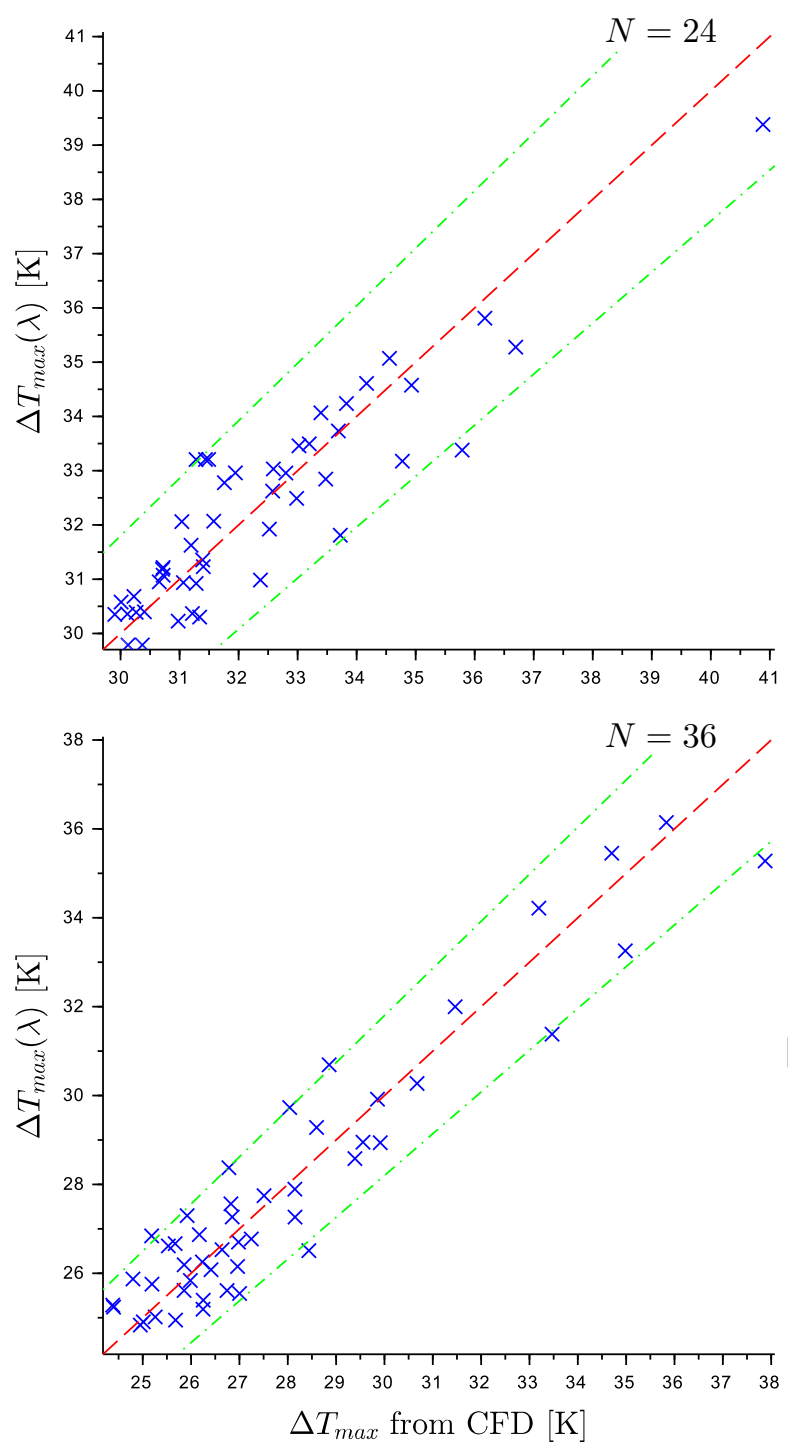

Figure 10: Parity graph for 24 (top) and 36 (bottom) heat sources. The temperature obtained with the CFD model is compared to the temperature predicted with the geometric parameter, $\lambda_{2}$. The green lines show a $\pm 6 \%$ error.

\section{Discussion}

The last distribution (number 21) illustrates the superiority of the proposed geometric parameter over the one found in $[23,25]$. With the formula suggested in those papers, this distribution would have been selected as the most efficient one. In fact, the parameters $\lambda_{1}, \lambda_{2}$ and $\lambda_{3}$ perform equally for a low number of sources. For high number of sources, the new parameters are much more suited.

Interestingly, the optimal parameter $b$ found in this study corresponds with the results presented in [16]. A parameter $b$ around 0.25 was obtained empirically which is what Silva and al. showed with a theoretical approach based on the assumption of a local Nusselt number proportional to $R a^{1 / 4}$.
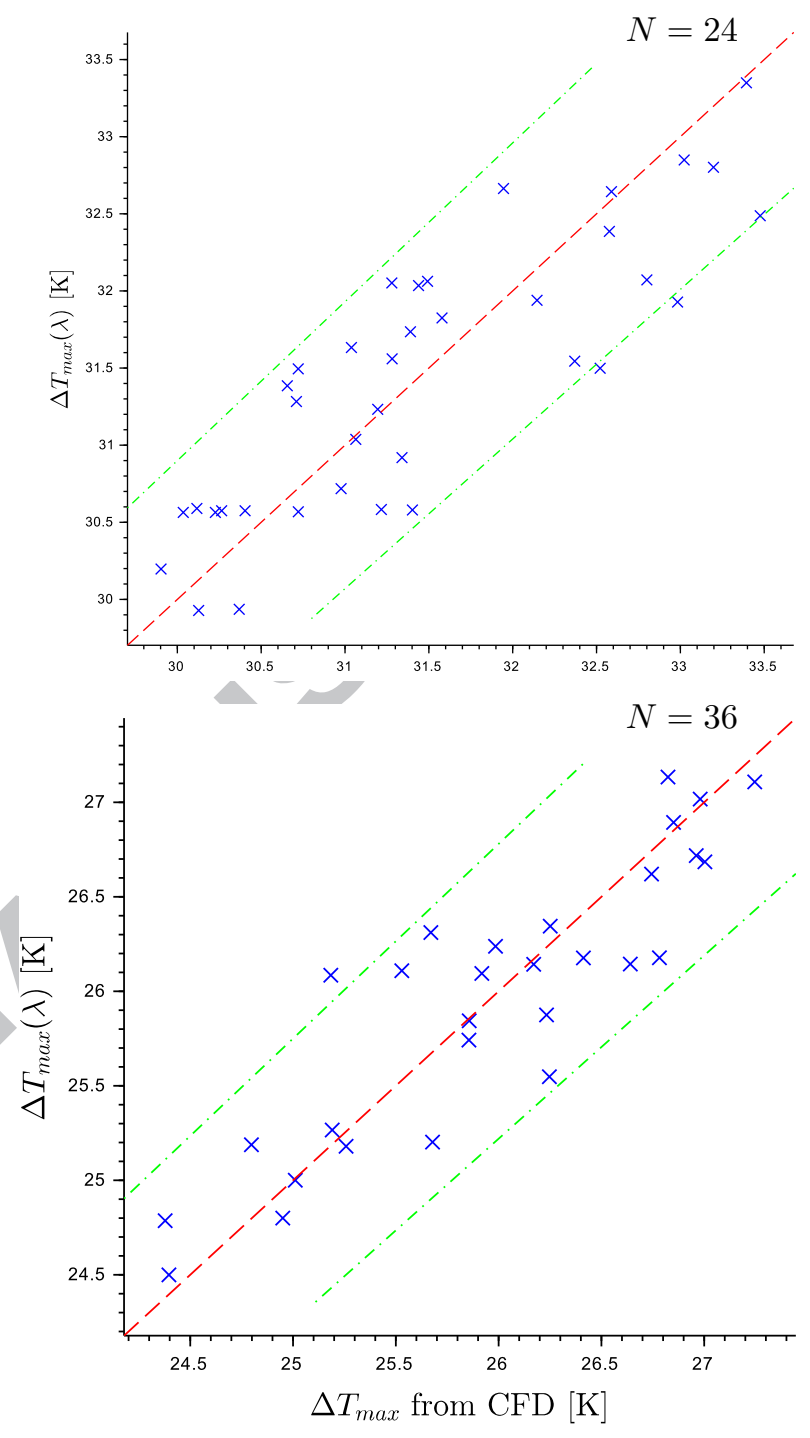

Figure 11: Parity graph 24 (top) and 36 (bottom) heat sources. The temperature obtained with the CFD model is compared to the temperature predicted with the geometric parameter, $\lambda_{3}$. The green lines show a $\pm 3 \%$ error.

Simpler distributions on regular grid might still be preferable in the manufacturing process. In such a case, the cooling capacity will be degraded by several percent, which might be a relevant choice. The more heat source density, the more significant this degradation will be. In such cases, vertical and horizontal spacings should be selected with care.

Distributions 1, 3, 5 and 7 are one example of a set of similar configurations differing only by the horizontal/vertical- spacing ratio. It appears that there exists an optimal aspect ratio for distribution on a regular grid. The same conclusion still holds true for other sets of similar distributions. This observation is in good agreement to the results presented in [37]. For the optimal staggered distribution, it can also be noticed that distribution number 15 for $N=24$ and distribution 17 for $N=36$ correspond 

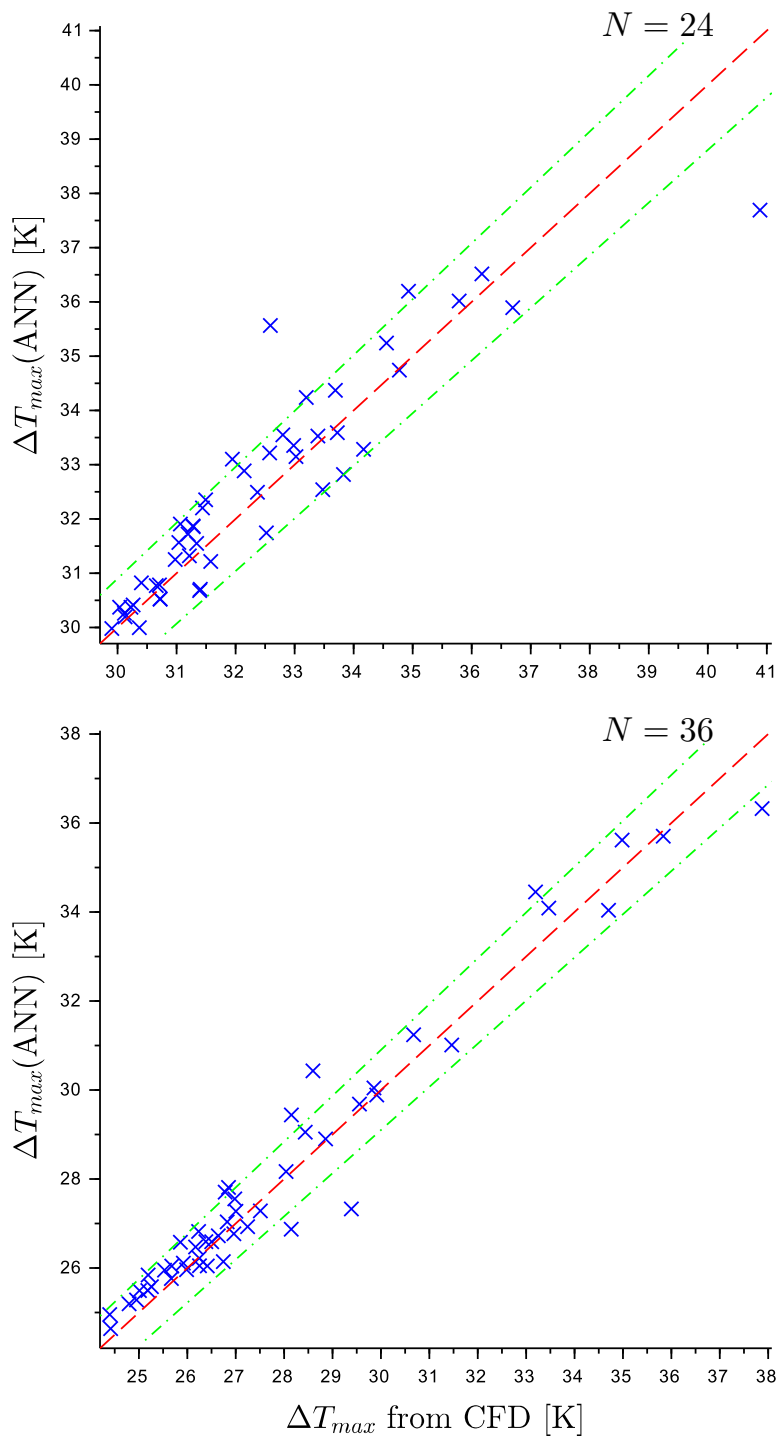

Figure 12: Parity graph 24 (top) and 36 (bottom) heat sources. The temperature obtained with the CFD model is compared to the temperature predicted with the ANN. The green lines show a $\pm 3 \%$ error.

both to an aspect ratio around 0.8 (see Figure 9).

The two proposed heuristic strategies do not depend on the area of $1 \times 2 \mathrm{~m}$ used in this study. The results are valid for the optimization of heatsink of different geometry.

A genetic algorithm (GA) approach was tried to find an optimal configuration, based the geometric parameter and the ANN. The results of this attempt indicated that the optima were already present among the first-guess distributions. Indeed, no better solution was obtained with the optimization algorithm even run repeated times.

\section{Conclusion}

The CFD model was validated with experimental data. Good agreement was shown for temperature profiles, local heat transfer.
The optimal (i.e. minimal temperature elevation for a given area and a given dissipated power) distribution was shown to be non uniform. In the vertical direction, the results confirmed earlier studies and a wider vertical spacing in the upper part of the plate should be preferred. In the horizontal direction, the distribution can be optimized by selecting a staggered configuration.

The effect of turbulence on the optimal spacing was represented by the model. When the flow transitions, an increasing vertical spacing is not optimal anymore.

Two strategies have been implemented in order to efficiently predict the temperature elevation for different distributions. Both a geometric parameter and an ANN were compared with the CFD model and showed good accuracy. They are two tools for rapid (computational time of $\approx 1$ day for CFD to $<0.01 \mathrm{~s}$ for heuristics) optimization of the distribution of any type of heat sources to be distributed on a vertical plate.

\section{Acknowledgment}

This work was supported by SweGRIDS, KIC InnoEnergy and StandUP for Energy.

[1] A. Baïri, E. Zarco-Pernia, J.-M. G. De María, A review on natural convection in enclosures for engineering applications. the particular case of the parallelogrammic diode cavity, Applied Thermal Engineering 63 (1) (2014) 304-322.

[2] Y. Cengel, A. Ghajar, Heat and Mass Transfer: Fundamentals and Applications, 5th Edition, McGraw Hill, 2015.

[3] E. Sanvicente, S. Giroux-Julien, C. Ménézo, H. Bouia, Transitional natural convection flow and heat transfer in an open channel, International Journal of Thermal Sciences 63 (2013) 87-104.

[4] A. Baudoin, C. Boström, M. Leijon, Thermal rating of a submerged substation for wave power, IEEE Transactions on Sustainable Energy 7 (1) (2016) 436-445.

[5] A. C. Kheirabadi, D. Groulx, Cooling of server electronics: A design review of existing technology, Applied Thermal Engineering 105 (2016) 622-638.

[6] S. Tou, C. Tso, X. Zhang, 3-d numerical analysis of natural convective liquid cooling of a $3 \times 3$ heater array in rectangular enclosures, International journal of heat and mass transfer 42 (17) (1999) 3231-3244.

[7] A. Baudoin, C. Boström, Thermal modelling of a passively cooled inverter for wave power, IET Renewable Power Generation 9 (4) (2015) 389-395.

[8] A. Bejan, Convection heat transfer, 4th Edition, John wiley \& sons, 2013.

[9] M. Mobedi, B. Sunden, Natural convection heat transfer from a thermal heat source located in a vertical plate fin, International communications in heat and mass transfer 33 (8) (2006) 943950.

[10] A. Müftüoğlu, E. Bilgen, Natural convection in an open square cavity with discrete heaters at their optimized positions, International Journal of Thermal Sciences 47 (4) (2008) 369-377.

[11] L. Boutina, R. Bessaïh, Numerical simulation of mixed convection air-cooling of electronic components mounted in an inclined channel, Applied Thermal Engineering 31 (11) (2011) 2052-2062.

[12] A. Da Silva, G. Lorenzini, A. Bejan, Distribution of heat sources in vertical open channels with natural convection, International Journal of Heat and Mass Transfer 48 (8) (2005) 1462-1469.

[13] T. Heindel, S. Ramadhyani, F. Incropera, Conjugate natural convection from an array of discrete heat sources: part 1two- 
and three-dimensional model validation, International Journal of Heat and Fluid Flow 16 (6) (1995) 501-510.

14] Q.-H. Deng, Fluid flow and heat transfer characteristics of natural convection in square cavities due to discrete source-sink pairs, International Journal of Heat and Mass Transfer 51 (25) (2008) 5949-5957.

[15] H. F. Öztop, P. Estellé, W.-M. Yan, K. Al-Salem, J. Orfi, O. Mahian, A brief review of natural convection in enclosures under localized heating with and without nanofluids, International Communications in Heat and Mass Transfer 60 (2015) 37-44.

[16] A. Da Silva, S. Lorente, A. Bejan, Optimal distribution of discrete heat sources on a wall with natural convection, International journal of heat and mass transfer 47 (2) (2004) 203-214.

[17] D. Saury, N. Rouger, F. Djanna, F. Penot, Natural convection in an air-filled cavity: Experimental results at large rayleigh numbers, International Communications in Heat and Mass Transfer 38 (6) (2011) 679-687.

18] R. Bessaih, M. Kadja, Turbulent natural convection cooling of electronic components mounted on a vertical channel, Applied thermal engineering 20 (2) (2000) 141-154.

[19] A. Bejan, S. Lorente, Design with constructal theory.

[20] M. Hajmohammadi, M. Rahmani, A. Campo, O. J. Shariatzadeh, Optimal design of unequal heat flux elements for optimized heat transfer inside a rectangular duct, Energy 68 (2014) 609-616.

[21] M. Hajmohammadi, S. Poozesh, M. Rahmani, A. Campo, Heat transfer improvement due to the imposition of non-uniform wall heating for in-tube laminar forced convection, Applied Thermal Engineering 61 (2) (2013) 268-277.

[22] M. Habib, S. Said, T. Ayinde, Characteristics of natural convection heat transfer in an array of discrete heat sources, Experimental Heat Transfer 27 (1) (2014) 91-111.

[23] T. Sudhakar, C. Balaji, S. Venkateshan, A heuristic approach to optimal arrangement of multiple heat sources under conjugate natural convection, International Journal of Heat and Mass Transfer 53 (1) (2010) 431-444.

[24] T. Hotta, C. Balaji, S. Venkateshan, Experiment driven annga based technique for optimal distribution of discrete heat sources under mixed convection, Experimental Heat Transfer 28 (3) (2015) 298-315.

[25] T. K. Hotta, S. P. Venkateshan, Optimal distribution of discrete heat sources under natural convection using ann-ga based technique, Heat Transfer Engineering 36 (2) (2015) 200-211.

[26] T. Dias, L. F. Milanez, Optimal location of heat sources on a vertical wall with natural convection through genetic algorithms, International Journal of Heat and Mass Transfer 49 (13) (2006) 2090-2096.

[27] A. K. da Silva, L. Gosselin, The numerical optimization of a neumann-type boundary for the graetz problem, Numerical Heat Transfer, Part A: Applications 59 (8) (2011) 577-593.

[28] R. R. Madadi, C. Balaji, Optimization of the location of multiple discrete heat sources in a ventilated cavity using artificial neural networks and micro genetic algorithm, International Journal of Heat and Mass Transfer 51 (9) (2008) 2299-2312.

[29] L. Daróczy, G. Janiga, D. Thévenin, Systematic analysis of the heat exchanger arrangement problem using multi-objective genetic optimization, Energy 65 (2014) 364-373.

[30] T. Sudhakar, C. Balaji, S. Venkateshan, Optimal configuration of discrete heat sources in a vertical duct under conjugate mixed convection using artificial neural networks, International Journal of Thermal Sciences 48 (5) (2009) 881-890.

[31] T. Sudhakar, A. Shori, C. Balaji, S. Venkateshan, Optimal heat distribution among discrete protruding heat sources in a vertical duct: a combined numerical and experimental study, Journal of Heat Transfer 132 (1) (2010) 011401.

[32] M. M. Mohamed, Air cooling characteristics of a uniform square modules array for electronic device heat sink, Applied Thermal Engineering 26 (5) (2006) 486-493.

[33] R. Bornoff, J. Parry, An additive design heatsink geometry topology identification and optimisation algorithm, in: Thermal
Measurement, Modeling \& Management Symposium (SEMITHERM), 2015 31st, IEEE, 2015, pp. 303-308.

[34] L. Exizidis, F. Vallée, Z. De Grève, J. Lobry, V. Chatziathanasiou, Thermal behavior of power cables in offshore wind sites considering wind speed uncertainty, Applied Thermal Engineering 91 (2015) 471-478.

[35] Y. Alexandrova, R. S. Semken, J. Pyrhönen, Permanent magnet synchronous generator design solution for large direct-drive wind turbines: Thermal behavior of the lc dd-pmsg, Applied Thermal Engineering 65 (1) (2014) 554-563.

[36] Z. Bocu, Z. Altac, Laminar natural convection heat transfer and air flow in three-dimensional rectangular enclosures with pin arrays attached to hot wall, Applied Thermal Engineering 31 (16) (2011) 3189-3195.

[37] A. Baudoin, D. Saury, B. Zhu, C. Boström, Experimental optimization of passive cooling of a heat source array flush-mounted on a vertical plate, Energies 9 (11) (2016) 912.

[38] A. Baudoin, D. Saury, Temperature and velocity measurements in a buoyant flow induced by a heat source array on a vertical plate, submitted to Experimental Thermal and Fluid Science.

[39] T. L. Bergman, F. P. Incropera, D. P. DeWitt, A. S. Lavine, Fundamentals of heat and mass transfer, John Wiley \& Sons, 2011. 
"Optimized distribution of a large number of power electronics components cooled by conjugate turbulent natural convection" - Highlights:

- Multiple power electronics modules are mounted on a plate with indirect liquid cooling.

- The distribution of up to 36 heat sources is optimized to enhance cooling.

- Turbulent effects are highlighted and influence the optimal vertical spacing.

- Use of geometric parameters and ANN are efficient alternatives for comparing layouts. 